\title{
异单叶大黄素及其类似结构茋类化合物的合成方法
}

\author{
赵剑阳 ${ }^{a, b}$ 郑紫华 ${ }^{a, b}$ 黄晴菲 ${ }^{a}$ 邓金根 ${ }^{c}$ 朱 槿*,a 王启卫*,a \\ $\left({ }^{a}\right.$ 中国科学院成都有机化学研究所 成都 610041) \\ $\left(^{b}\right.$ 中国科学院大学 北京 100049) \\ $\left({ }^{c}\right.$ 四川大学华西药学院 成都 610041)
}

\begin{abstract}
摘要 发展了一种异单叶大黄素及其类似结构的芪类化合物的合成方法. 以 3,5-二差基苯甲酸为起始原料, 通过苄基 保护酚羟基, Wittig-Horner 反应构建反式二苯乙烯结构产物, 在钯碳/甲酸铵条件下, 以较高收率脱芐基得到异单叶大 黄素, 总收率高达 $48 \%$. 并将该催化体系成功地应用于一系列羟基芪类化合物的合成, 取得良好的效果. 各步反应收 率高, 条件温和, 所用试剂成本低、毒性小, 操作简单、安全, 适合较大量生产. 为羟基茋类化合物的大批量合成提供 了简便的途径.
\end{abstract}

关键词 异单叶大黄素; 羟基茋类化合物; 钯碳/甲酸铵; 脱苄基

\section{A Method for the Synthesis of the Isorhapontigenin and the Stilbene Compounds in Similar Structures}

\author{
Zhao, Jianyang ${ }^{a, b}$ \\ Zheng, Zihua ${ }^{a, b}$ \\ Huang, Qingfei ${ }^{a}$ \\ Deng, Jingen ${ }^{c}$ \\ Zhu, Jin *,a \\ Wang, Qiwei*,a \\ $\left({ }^{a}\right.$ Chengdu Institute of Organic Chemistry, Chinese Academy of Science, Chengdu 610041) \\ $\left({ }^{b}\right.$ University of Chinese Academy of Sciences, Beijing 100049) \\ ( ${ }^{c}$ West China School of Pharmacy Sichuan University, Chengdu 610041)
}

\begin{abstract}
A method for the synthesis of the isorhapontigenin and the stilbene compounds in similar structures was developed. Using 3,5-dihydroxybenzoic acid as starting material, benzyl protecting the phenolic hydroxyl, via the Wittig-Horner reaction to construct the trans stilbene structure, and then debenzylation under the palladium on carbon/ammonium formate system condition, the isorhapontigenin could be obtained in the yield of up to $48 \%$. The catalytic system was successfully applied in the synthesis of a series of hydroxyl stilbene compounds, which could achieve good results. All the reactions worked under mild conditions and obtained in good yield. Furthermore the materials were of cheap and less toxicity. It provided us a simple way to synthesize hydroxyl stilbene compounds in large scale.
\end{abstract}

Keywords isorhapontigenin; hydroxyl stilbene compounds; palladium on carbon/ammonium formate; debenzylation

异单叶大黄素(Isorhapontigenin, $(E)-3,4$ ',5-trihydroxy-3'-methoxystilbene)是近年来备受关注的天然羟基茋 类化合物白蔡芦醇的 $3^{\prime}$-位甲氧基化衍生物, 具有多种 生物活性. 如比维生素 $\mathrm{E}$ 更强的体外抗氧化作用 ${ }^{[1,2]}$, 白 血病细胞中的抗过敏作用 ${ }^{[3]}$, 心脏保护作用 ${ }^{[4]}$, 抗病毒 作用 ${ }^{[5]}$, 能抑制膀胱癌细胞增殖, 促进化疗药物效 果 ${ }^{[6]}$, 抑制人肝癌 $\mathrm{HepG} 2$ 细胞的增殖及诱导的 $\mathrm{HepG} 2$ 细胞调亡 ${ }^{[7]}$, 对心肌肥厚有着良好的抑制作用, 有望在
抗肿瘤、抗衰老、抗心血管疾病等领域获得广泛应用. 异 单叶大黄素主要从植物中提取，其来源十分有限，因此 研究其化学合成具有重要意义. 目前, 异单叶大黄素的 合成方法报道较少，制备成本较高，副产物较多，分离 困难 ${ }^{[8]}$.

Bieg 等 ${ }^{[9]}$ 报道了通过钯碳/甲酸铵氢转移选择性地 脱茮基得到糖苷类化合物的方法. 本文将钯碳甲酸铵 催化脱茮基的方法用于脱去芐基保护的异丹叶大黄素

\footnotetext{
*E-mail: wqw@cioc.ac.cn,jinzhu@cioc.ac.cn

Received August 27, 2015; revised October 31, 2015; published online November 16, 2015.

Project supported by the Award for Innovative Talents in Chengdu High-tech Zone.

成都市高新区创新创业人才奖资助项目.
} 
上的苄基以合成异单叶大黄素, 有效地提高了异丹叶 大黄素的产率，并将该方法应用到系列茋类化合物的 合成.

\section{1 结果与讨论}

\section{1 脱苄基反应条件优化}

我们以 3,5 -二羟基苯甲酸或者没食子酸为原料，根 据 Thakkar 等 ${ }^{[10]}$ 报道方法经苄基保护、甲酯化、四氢锂 铝还原、溴代和 Witting-Horner 反应得到系列中间体化
合物 7a 7f (Scheme 1), 并以化合物 7a 为底物研究了钯 碳/甲酸铵氢转移催化脱茮基的反应条件. 考察了反应 溶剂、钯碳催化剂的用量、甲酸铵用量、反应温度和反 应时间对反应的影响. 结果如表 1 所示.

从表 1 中(Entries 1 4)可以看出, 1,2-二氯乙烷与甲 醇体积比为 $1 / 1$ 时, 脱茮基反应效果最好, 并且发现溶 剂体系中随着甲醇比例的减少, 反应速度加快, 所得产 物中双键被还原的副产物 1as 含量增加, 反应溶剂中甲 醇对产物双键被还原具有一定的抑制作用. 从表 1 中

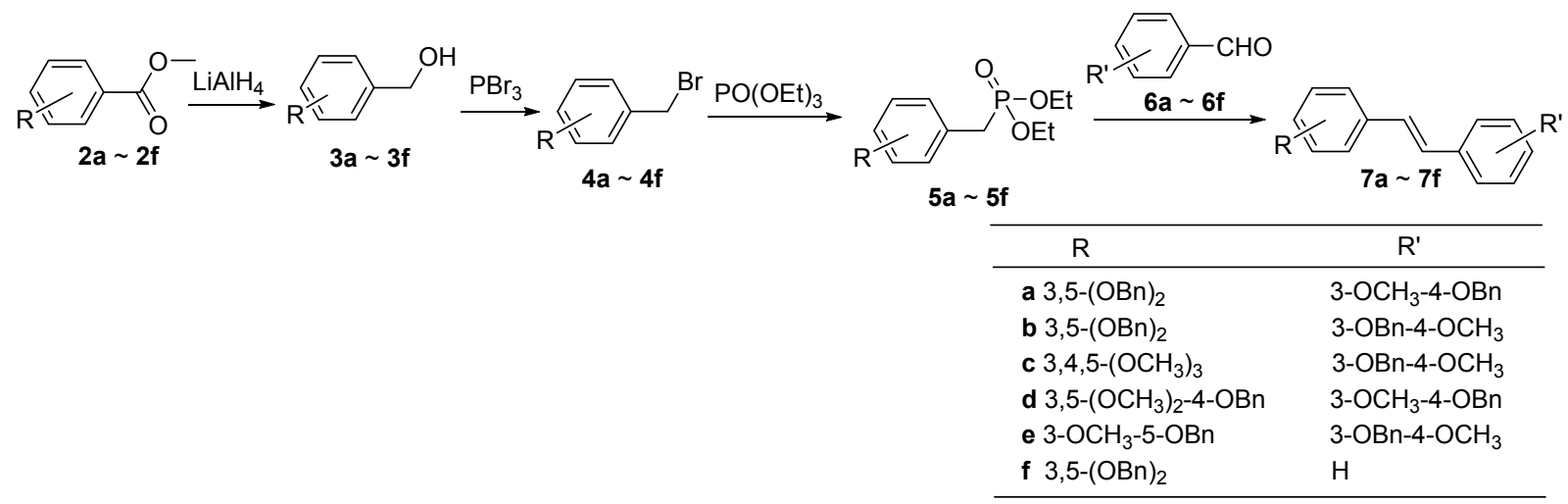

图式 1 化合物 $7 \mathbf{a} \sim 7 \mathbf{f}$ 的合成

Scheme 1 Synthesis of compound $7 \mathbf{a} \sim 7 \mathbf{f}$

表 1 化合物 7a 的脱苄基反应条件优化 ${ }^{a}$

Table 1 Optimization of the conditions for debenzylation of compound $7 \mathbf{a}$
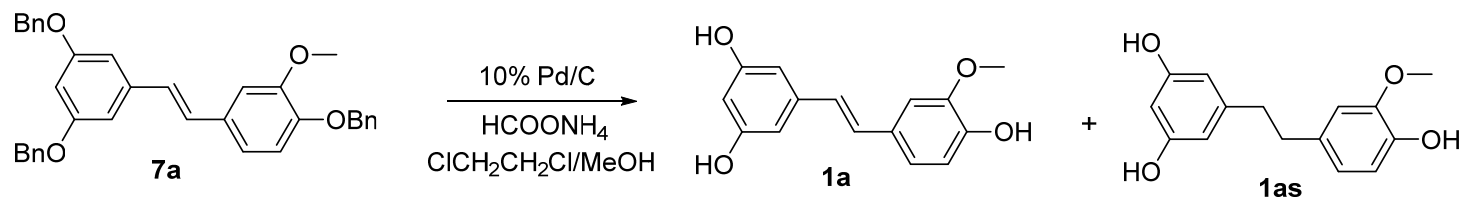

\begin{tabular}{|c|c|c|c|c|c|c|}
\hline Entry & $w$ (钯碳) $/ \%$ & $x$ (甲酸铵) & $V(1,2$-二氯乙烷) $： V$ (甲醇 $)$ & 温度 $/{ }^{\circ} \mathrm{C}$ & 时间 $/ \mathrm{min}$ & 收率 ${ }^{b} / \%$ \\
\hline 1 & 20 & 6.0 & $0 / 3$ & 40 & 40 & Trace \\
\hline 2 & 20 & 6.0 & $1 / 2$ & 40 & 40 & 14 \\
\hline 3 & 20 & 6.0 & $1.5 / 1.5$ & 40 & 40 & 45 \\
\hline 4 & 20 & 6.0 & $1.8 / 1.2$ & 40 & 40 & 16 \\
\hline 5 & 10 & 6.0 & $1.5 / 1.5$ & 40 & 40 & 11 \\
\hline 6 & 30 & 6.0 & $1.5 / 1.5$ & 40 & 40 & 41 \\
\hline 7 & 20 & 5.0 & $1.5 / 1.5$ & 40 & 40 & Trace \\
\hline 8 & 20 & 7.0 & $1.5 / 1.5$ & 40 & 40 & 35 \\
\hline 9 & 20 & 6.0 & $1.5 / 1.5$ & 50 & 40 & 31 \\
\hline 10 & 20 & 6.0 & $1.5 / 1.5$ & 30 & 40 & 34 \\
\hline 11 & 20 & 6.0 & $1.5 / 1.5$ & 20 & 40 & 30 \\
\hline 12 & 20 & 6.0 & $1.5 / 1.5$ & 40 & 35 & 51 \\
\hline 13 & 20 & 6.0 & $1.5 / 1.5$ & 40 & 30 & 64 \\
\hline 14 & 20 & 6.0 & $1.5 / 1.5$ & 40 & 25 & 72 \\
\hline 15 & 20 & 6.0 & $1.5 / 1.5$ & 40 & 20 & 46 \\
\hline $16^{c}$ & 20 & 6.0 & $15 / 15$ & 40 & 25 & 67 \\
\hline $17^{d}$ & 20 & 6.0 & $75 / 75$ & 40 & 25 & 68 \\
\hline
\end{tabular}


Entries 3,5 和 6 可以看出, 钯碳用量过少, 反应速度较 慢, 钯碳用量太大, 反应速度过快而容易产生副产物 1as, 钯碳用量为原料 7a 质量的 $20 \%$ 时, 脱茮基反应效 果最好. 结合表 1 中 Entries 3, 7 和 8 可以看出, 甲酸铵 的用量过少, 反应速度慢, 未完全脱苄基副产物较多, 甲酸铵用量太大, 则反应速度过快, 且 1 as 增多, 当甲 酸铵用量为 6 equiv. 时, 脱芐基反应效果最好. 从表 1 中 Entries 9 11) 可以看出温度对反应影响相对较小, $40{ }^{\circ} \mathrm{C}$ 反应相对较好. 接着, 我们对反应时间进行了研 究, 从表 1 中 Entries $12 \sim 15$ 可以看出, 反应时间过长, 更容易得到 1as, $25 \mathrm{~min}$ 时, 得到目标产物收率最高. 在 此条件下, 我们对反应底物进行 1 和 5 克级放大反应研 究, 从表 1 中 Entries 16, 17 可以看出, 该催化体系适合 异丹叶大黄素的较大批量制备.

\section{2 脱苄基反应化学选择性研究}

Ranu 等 ${ }^{[11]}$ 报道, 钯碳甲酸铵催化体系能选择性还 原 $\alpha, \beta$-不饱和酮上的共轭碳碳双键, 因此, 在上述脱茮 基反应过程中, 会产生部分碳碳双键被还原副产物 1 as. 从表 1 中 Entries 12,13 可以看出, 反应时间对产物收率 具有一定的影响. 另外还发现随着时间的增加, 1as 也进 一步增加. 为了进一步研究反应时间对该反应的影响, 我们系统研究了 $1 \mathrm{a}$ 和 $1 \mathrm{as}$ 的收率和反应时间的关系(图 1). 从图 1 中可以看出, $\mathbf{1 a}$ 收率在一定时间 $(23 \mathrm{~min}$ 左右) 达到峰值后逐渐下降, 1as 则随反应的进行逐渐增加. 从 而, 得出通过对反应时间的控制, 可以较高收率的选择 性得到目标产物.

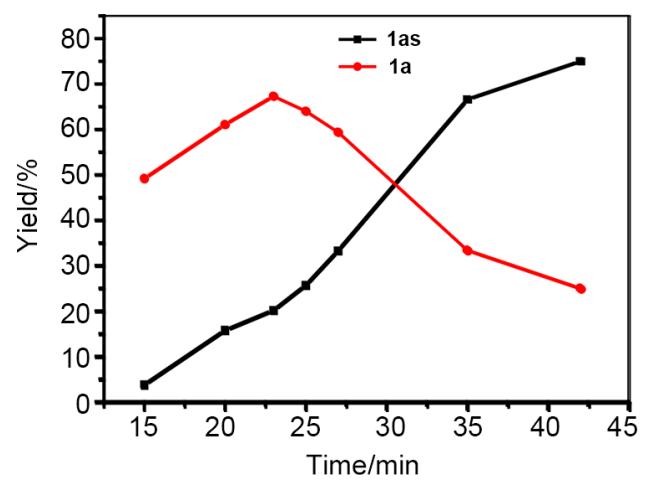

图 1 脱苄基反应中 $1 \mathrm{a}$ 和 1 as 收率对时间关系曲线

Figure 1 The curve of the yield of 1a-1as-reaction time

\section{3 不同底物的脱苄基反应}

在上述优化条件基础上, 对于不同的底物结构, 对 催化剂用量、溶剂、甲酸铵用量和反应时间进行适当的 调整, 进一步考查此催化体系的底物普适性(表 2). 对于 不同的底物 $7 \mathbf{b} \sim 7 \mathbf{f}$ 取得了 $40 \% \sim 85 \%$ 的收率, 进一步说 明了该体系具有较强的普适性.
表 2 不同底物的脱苄基反应 ${ }^{a}$

Table 2 The debenzylation of different substrates
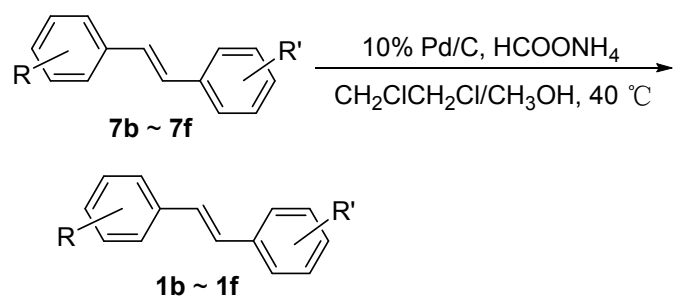

\begin{tabular}{ll}
\hline \multicolumn{1}{c}{$\mathrm{R}$} & \multicolumn{1}{c}{$\mathrm{R}^{\prime}$} \\
\hline b 3,5-(OH $)_{2}$ & $3-\mathrm{OH}-4-\mathrm{OCH}_{3}$ \\
c 3,4,5-(OCH$)_{3}$ & $3-\mathrm{OH}-4-\mathrm{OCH}_{3}$ \\
d 3,5-(OCH$)_{2}-4-\mathrm{OH}$ & $3-\mathrm{OCH}_{3}-4-\mathrm{OH}$ \\
e 3-OCH $-5-\mathrm{OH}$ & $3-\mathrm{OH}-4-\mathrm{OCH}_{3}$ \\
f 3,5-(OH $)_{2}$ & $\mathrm{H}$ \\
\hline
\end{tabular}

\begin{tabular}{|c|c|c|c|c|c|}
\hline Entry & 产物 & $w$ (钯碳) $/ \%$ & $x$ (甲酸铵) & 时间 $/ \mathrm{min}$ & 收率 ${ }^{b} / \%$ \\
\hline 1 & $1 b$ & 20 & 6.0 & 30 & 70 \\
\hline 2 & 1c & 10 & 4.0 & 30 & 85 \\
\hline 3 & 1d & 20 & 5.5 & 30 & 77 \\
\hline 4 & $1 \mathrm{e}$ & 10 & 5.5 & 30 & 73 \\
\hline 5 & 1f & 20 & 5.5 & 240 & 40 \\
\hline
\end{tabular}

\section{2 结论}

本文以廉价 3,5-二羟基苯甲酸或者没食子酸为起始 原料，通过苄基保护酚羟基，Wittig-Horner 反应构建系 列反式二苯乙烯结构. 并利用钯碳/甲酸铵催化脱苄基 高效制备异单叶大黄素及其类似结构的茋类化合物. 发 现通过对反应时间的控制可以有效控制双键被还原的 副产物。该方法反应条件温和，所用试剂毒性小，操作 简单安全，可控，更易用于该类化合物的大量制备.

\section{3 实验部分}

\section{1 仪器与试剂}

熔点由 BUCHI 公司 B-545 型熔点仪测定; ${ }^{1} \mathrm{H}$ NMR 和 ${ }^{13} \mathrm{C}$ NMR 由 Bruker-300 型核磁共振仪测定，三甲基硅 烷为内标; 质谱以 Agilent $6460 \mathrm{MS}(50 \mathrm{eV})$ 型质谱仪测 定; 薄层层析(TLC)和柱层析分别使用山东青岛海洋化 工厂生产的薄层层析硅胶 GF254 型硅胶和 300 400 目 柱层析硅胶. 实验中所用原料化合物均为分析纯. 钯碳 催化剂来自阿尔法试剂公司生产的 A12012 型含钯 10\% 的钯碳.

化合物 2a $2 \mathbf{f}$ 的制备分别参考文献[12 14]方法, 化合物 7a $\sim 7 \mathbf{f}$ 的全合成参考文献 $[8,10]$ 方法, 波谱数据 与文献值一致. 


\section{2 实验方法}

\subsection{1 异单叶大黄素(1a)的合成}

将化合物 7a $106 \mathrm{mg}(0.2 \mathrm{mmol})$ 和甲酸铵 $76 \mathrm{mg}(1.2$ $\mathrm{mmol})$ 溶于 $3 \mathrm{~mL}$ 混合溶剂 $[V(1,2-$ 二氯乙烷 $): V($ 甲 醇 $)=1 / 1$ ]中, 加热 $40{ }^{\circ} \mathrm{C}$, 搅拌 $15 \mathrm{~min}$, 原料溶解. 保温

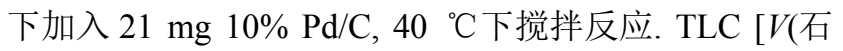
油醚 $) / V($ 乙酸乙酯 $)=2 / 1$ ] 监测至反应完全, 约 $25 \mathrm{~min}$. 过滤除去钯碳, 水洗母液以除去无机盐, 减压蒸除母液 溶剂, 残留物经柱层析 $[V$ (石油醚 $) / V($ 乙酸乙酯 $)=3 / 1]$ 得 到 $37 \mathrm{mg}$ 淡黄色固体, 收率 $72 \%$. m.p. $170 \sim 174{ }^{\circ} \mathrm{C}$ (文 献值 $\left.{ }^{88]} 170 \sim 173{ }^{\circ} \mathrm{C}\right) ;{ }^{1} \mathrm{H}$ NMR $\left(300 \mathrm{MHz}, \mathrm{CD}_{3} \mathrm{COCD}_{3}\right)$ $\delta: 8.23$ (s, $2 \mathrm{H}, \mathrm{OH}, \mathrm{D}_{2} \mathrm{O}$ exchangeable), $7.71(\mathrm{~s}, 1 \mathrm{H}, \mathrm{OH}$, $\mathrm{D}_{2} \mathrm{O}$ exchangeable), $7.18(\mathrm{~d}, J=1.8 \mathrm{~Hz}, 1 \mathrm{H}), 7.03 \sim 6.97$ (m, 2H), $6.90(\mathrm{~d}, J=16.3 \mathrm{~Hz}, 1 \mathrm{H}), 6.79(\mathrm{~d}, J=8.1 \mathrm{~Hz}$, $1 \mathrm{H}), 6.53(\mathrm{~d}, J=2.0 \mathrm{~Hz}, 2 \mathrm{H}), 6.26(\mathrm{t}, J=2.1 \mathrm{~Hz}, 1 \mathrm{H}), 3.86$ $(\mathrm{s}, 3 \mathrm{H}) ;{ }^{13} \mathrm{C}$ NMR $\left(75 \mathrm{MHz}, \mathrm{CD}_{3} \mathrm{COCD}_{3}\right) \delta: 159.5,148.5$, $147.5,140.8,130.4,129.4,127.0,121.1,115.9,110.1$, 105.7, 102.7, 56.2; ESI-MS $m / z$ : $259.1\left([\mathrm{M}+\mathrm{H}]^{+}\right)$.

\subsection{2 化合物 $\mathbf{1 b} \sim \mathbf{1 f}$ 的合成}

将化合物 $7 \mathbf{b} \sim 7 \mathbf{f}(0.2 \mathrm{mmol})$ 和甲酸铵 $(1.2 \mathrm{mmol})$ 溶 于 $3 \mathrm{~mL}$ 混合溶剂 $[V(1,2$-二氯乙烷) $: V$ (甲醇 $)=1 / 2]$ 中, 加热 $40{ }^{\circ} \mathrm{C}$, 搅拌 $15 \mathrm{~min}$, 原料溶解. 保温下加入质量 分数 $10 \% \sim 20 \%$ 的 $10 \% \mathrm{Pd} / \mathrm{C}, 40{ }^{\circ} \mathrm{C}$ 下搅拌反应, TLC $[V($ 石油醚 $) / V($ 乙酸乙酯 $)=2 / 1]$ 监测至反应完全, 具体数 据如表 2 所示. 过滤除去钯碳, 水洗母液以除去无机盐, 减压蒸除母液溶剂, 残留物经柱层析 $[V($ 石油醚 $) / V($ 乙酸 乙酯) $=3 / 1$ ] 得到产物 $\mathbf{1 b} \sim \mathbf{1 f}$.

单叶大黄素/ $(E)-3,5,4^{4}$,-三甲氧基-3'-羟基二苯乙烯 (1b): $36 \mathrm{mg}$ 类白色固体, 收率 $70 \%$. m.p. $180 \sim 184{ }^{\circ} \mathrm{C}$ (文献值 ${ }^{[15]} 184 \sim 188{ }^{\circ} \mathrm{C}$ ); ${ }^{1} \mathrm{H}$ NMR $(300 \mathrm{MHz}$, $\left.\mathrm{CD}_{3} \mathrm{COCD}_{3}\right) \delta: 8.22$ (s, $2 \mathrm{H}, \mathrm{OH}, \mathrm{D}_{2} \mathrm{O}$ exchangeable), 7.60 (s, $1 \mathrm{H}, \mathrm{OH}, \mathrm{D}_{2} \mathrm{O}$ exchangeable), $7.10(\mathrm{~d}, J=2.0 \mathrm{~Hz}, 1 \mathrm{H})$, $7.02 \sim 6.86(\mathrm{~m}, 4 \mathrm{H}), 6.56(\mathrm{~d}, J=2.1 \mathrm{~Hz}, 2 \mathrm{H}), 6.28(\mathrm{t}, J=$ $2.1 \mathrm{~Hz}, 1 \mathrm{H}), 3.85(\mathrm{~s}, 3 \mathrm{H}) ;{ }^{13} \mathrm{C}$ NMR $(75 \mathrm{MHz}$, $\left.\mathrm{CD}_{3} \mathrm{COCD}_{3}\right) \delta: 159.6,148.3,147.6,140.7,131.7,129.1$, 127.6, 119.7, 113.3, 112.4, 105.7, 102.8, 56.3; ESI-MS $\mathrm{m} / z: 259\left([\mathrm{M}+\mathrm{H}]^{+}\right)$. 表征数据与参考文献 $[16]$ 对应.

(E)-3,4,5,4',-四甲氧基-3'-羟基二苯乙烯(1c): $63 \mathrm{mg}$ 蜡状产物, 收率 $85 \% .{ }^{1} \mathrm{H}$ NMR (300 MHz, DMSO- $d_{6}$ ) $\delta$ : 9.01 (s, 1H, OH, $\mathrm{D}_{2} \mathrm{O}$ exchangeable), 7.07 (d, $J=16.3 \mathrm{~Hz}$, $1 \mathrm{H}), 7.02 \sim 6.89(\mathrm{~m}, 4 \mathrm{H}), 6.87(\mathrm{~s}, 2 \mathrm{H}), 3.82(\mathrm{~s}, 6 \mathrm{H}), 3.78$ (s, $3 \mathrm{H}), 3.66(\mathrm{~s}, 3 \mathrm{H}) ;{ }^{13} \mathrm{C} \mathrm{NMR}\left(75 \mathrm{MHz}, \mathrm{CDCl}_{3}\right) \delta: 153.3$, $146.4,145.8,137.6,133.3,130.9,127.8,127.0,119.2$, 111.7, 110.6, 103.3, 60.9, 56.1, 56.0; ESI-MS m/z: 317.1 $\left([\mathrm{M}+\mathrm{H}]^{+}\right)$. 表征数据与参考文献 $[17]$ 对应.
(E)-3,5,3',-三甲氧基-4,4'-二羟基二苯乙烯(1d)：46 $\mathrm{mg}$ 类白色固体，收率 $77 \%$. m.p. $154 \sim 157{ }^{\circ} \mathrm{C}$ (文献值 ${ }^{[18]}$ $\left.156 \sim 159{ }^{\circ} \mathrm{C}\right) ;{ }^{1} \mathrm{H}$ NMR $\left(300 \mathrm{MHz}, \mathrm{CD}_{3} \mathrm{COCD}_{3}\right) \delta: 7.65$ (s, $1 \mathrm{H}, \mathrm{OH}, \mathrm{D}_{2} \mathrm{O}$ exchangeable), $7.27\left(\mathrm{~s}, 1 \mathrm{H}, \mathrm{OH}, \mathrm{D}_{2} \mathrm{O}\right.$ exchangeable), 7.18 (d, $J=1.9 \mathrm{~Hz}, 1 \mathrm{H}), 7.04$ (d, $J=16.4$ Hz, 1H), 6.98 (d, $J=16.3 \mathrm{~Hz}, 1 \mathrm{H}), 6.97$ (d, $J=1.9 \mathrm{~Hz}$, $1 \mathrm{H}), 6.86(\mathrm{~s}, 2 \mathrm{H}), 6.80(\mathrm{~d}, J=8.1 \mathrm{~Hz}, 1 \mathrm{H}), 3.89(\mathrm{~s}, 3 \mathrm{H})$, $3.87(\mathrm{~s}, 6 \mathrm{H}) ;{ }^{13} \mathrm{C}$ NMR $\left(75 \mathrm{MHz}\right.$, DMSO- $\left.d_{6}\right) \delta: 148.2$, $147.9,146.2,135.2,129.2,128.1,126.2,126.1,119.6$, 115.6, 109.5, 103.8, 56.0, 55.6; ESI-MS m/z: 303.1 ([M+ $\mathrm{H}]^{+}$). 表征数据与参考文献[18]对应.

(E)-3,4',-二甲氧基-3',5-二羟基二苯乙烯(1e): $39 \mathrm{mg}$ 类白色固体, 收率 $73 \%$. m.p. $159 \sim 162{ }^{\circ} \mathrm{C} ;{ }^{1} \mathrm{H}$ NMR $\left(300 \mathrm{MHz}, \mathrm{DMSO}-d_{6}\right) \delta: 9.42\left(\mathrm{~s}, 1 \mathrm{H}, \mathrm{OH}, \mathrm{D}_{2} \mathrm{O}\right.$ exchangeable), 8.99 (s, $1 \mathrm{H}, \mathrm{OH}, \mathrm{D}_{2} \mathrm{O}$ exchangeable), $7.02 \sim 6.95(\mathrm{~m}$, $3 \mathrm{H}), 6.90(\mathrm{~d}, J=8.4 \mathrm{~Hz}, 1 \mathrm{H}), 6.84(\mathrm{~d}, J=16.4 \mathrm{~Hz}, 1 \mathrm{H})$, $6.58(\mathrm{~s}, 1 \mathrm{H}), 6.53(\mathrm{~s}, 1 \mathrm{H}), 6.22(\mathrm{t}, J=2.1 \mathrm{~Hz}, 1 \mathrm{H}), 3.78(\mathrm{~s}$, $3 \mathrm{H}), 3.72$ (s, $3 \mathrm{H}) ;{ }^{13} \mathrm{C}$ NMR (75 MHz, DMSO- $d_{6}$ ) $\delta: 160.6$, $158.6,147.7,146.6,139.3,130.1,128.5,126.3,118.5$, 113.0, 112.2, 106.0, 102.7, 100.6, 55.7, 55.0; ESI-MS $\mathrm{m} / \mathrm{z}$ : $273.1\left([\mathrm{M}+\mathrm{H}]^{+}\right)$.

银松素 $/(E)-3,5$-二羟基二苯乙烯(1f): $17 \mathrm{mg}$ 浅黄色 固体, 收率 $40 \%$. m.p. $153 \sim 157{ }^{\circ} \mathrm{C}$ (文献值 ${ }^{[10]} 156 \sim$ $\left.159{ }^{\circ} \mathrm{C}\right) ;{ }^{1} \mathrm{H}$ NMR $\left(300 \mathrm{MHz}, \mathrm{CD}_{3} \mathrm{COCD}_{3}\right) \delta: 8.30(\mathrm{~s}, 2 \mathrm{H}$, $\mathrm{OH}, \mathrm{D}_{2} \mathrm{O}$ exchangeable), $7.58(\mathrm{~m}, 2 \mathrm{H}), 7.38 \sim 7.33(\mathrm{~m}$, $2 \mathrm{H}), 7.28 \sim 7.22(\mathrm{~m}, 1 \mathrm{H}), 7.09$ (s, 2H), $6.59(\mathrm{~d}, J=2.1 \mathrm{~Hz}$, $2 \mathrm{H}), 6.31$ (t, $J=2.1 \mathrm{~Hz}, 1 \mathrm{H}) ;{ }^{13} \mathrm{C}$ NMR $(75 \mathrm{MHz}$, $\left.\mathrm{CD}_{3} \mathrm{COCD}_{3}\right) \delta: 159.6,140.3,138.4,129.8,129.5,129.1$, 128.3, 127.3, 106.0, 103.2; ESI-MS $m / z: 213.1\left([\mathrm{M}+\mathrm{H}]^{+}\right)$. 表征数据与参考文献 $[10,19]$ 对应.

辅助材料(Supporting Information) 目标化合物 1a 1f 的 ${ }^{1} \mathrm{H}$ NMR、 ${ }^{13} \mathrm{C}$ NMR 及部分区域放大的 ${ }^{1} \mathrm{H}$ NMR 图 谱, 目标化合物 $1 \mathrm{a} \sim \mathbf{1 f}$ 经重水交换后的 ${ }^{1} \mathrm{H} N \mathrm{NMR}$ 图谱数 据. 这些材料可以免费从本刊网站(http://sioc-journal. $\mathrm{cn} /$ )上下载.

\section{References}

[1] Wang, Q. L.; Lin, M.; Liu, G. T. Chin. Pharm. J. 2001, 36, 810 (in Chinese).

(王庆利, 林茂, 刘耕陶, 中国药学杂志, 2001, 36, 810.)

[2] Iliya, I.; Ali, Z.; Tanaka, T.; Iinuma, M.; Furusawa, M.; Nakaya, K.-I.; Murata, J.; Darnaedi, D.; Matsuura, N.; Ubukata, M. Phytochemistry 2003, 62, 601 .

[3] Matsuda, H.; Tewtrakul, S.; Morikawa, T.; Yoshikawa, M. Bioorg. Med. Chem. 2004, 12, 4871.

[4] Li, H.-L.; Wang, A.-B.; Huang, Y.; Liu, D.-P.; Wei, C.; Williams, G. M. Free Radical Biology Med. 2005, 38, 243. 
[5] Liu, A.-L.; Yang, F.; Zhu, M.; Zhou, D.; Lin, M.; Lee, S. M.-Y. Planta Med. 2010, 76, 1874.

[6] Fang,Y.; Hou, Q.; Pang, H.-M. Chin. Pharm. J. 2014, 49, 653 (in Chinese).

(方勇, 侯琦, 潘宏铭, 中国药学杂志, 2014, 49, 653.)

[7] He, P.; Liu, Z.-M.; Liao, Z.-Y. Anat. Res. 2005, 27, 279 (in Chinese).

(何平, 刘振明, 廖振裕, 解剖学研究, 2005, 27, 279.)

[8] Li, W.-L.; He, K.-K.; Li, Y.; Hou, Z.-J. Acta Chim. Sinica 2005, 63, 1607 (in Chinese).

(李文玲，何㑆㑆，李瀛，侯自杰，化学学报, 2005, 63, 1607.)

[9] Bieg, T.; Szeja, W. Synth. Commun. 1985, 15, 76.

[10] Thakkar, K.; Geahlen, R. L.; Cushman, M. J. Med. Chem. 1993, 36, 2950.

[11] Ranu, B. C.; Sarkar, A. Tetrahedron Lett. 1994, 35(46), 8649.

[12] Negi, A. S.; Darokar, M.-P.; Chattapodhyay, S.-K.; Grag, A.;
Bhattacharya, A.-K.; Srivastava, V.; Khanuja, S. P. S. Bioorg. Med. Chem. Lett. 2005, 15, 1243.

[13] Negi, A. S.; Chattapodhyay, S.-K.; Srivastava, S.; Bhattacharya, A.-K. Synth. Commun. 2005, 35, 15.

[14] Koolaji, N.; Abdallah, A. M.; Tran, V. H.; Duke, R. K.; Duke, C. C. Eur. J. Med. Chem. 2013, 63, 415.

[15] Kim, D.-H.; Park, E.-K.; Bae, E.-A.; Han, M.-J. Biol. Pharm. Bull. 2000, 23(7), 830.

[16] Lee, H.-S.; Lee, B.-W.; Kim, M.-R.; Jun, J.-G. Bull. Korean Chem. Soc. 2010, 31(4), 971.

[17] Zhou, Y.; Huang, Q.; Huang, T.-K.; Ni, Q.-C.; Zhang, E.-S.; Xu, T.-L.; Yuan, M.; Li, J. Org. Biomol. Chem. 2013, 11, 6967.

[18] Lonsky, L.; Lonsky, W.; Kratzl, K. Monatsh. Chem. 1976, 107, 685.

[19] Cheng, J.-C.; Fang, J.-G.; Chen, W.-F.; Zhou, B.; Yang, L.; Liu, Z.-L. Bioorg. Chem. 2006, 34, 142. 